\title{
Lumen
}

Selected Proceedings from the Canadian Society for Eighteenth-Century Studies

\section{L'ange et le cadavre puant : Doctrine et culte des anges en France au XVIII ${ }^{\mathrm{e}}$ siècle}

\section{Nancy Senior}

Volume 15, 1996

URI : https://id.erudit.org/iderudit/1012481ar

DOI : https://doi.org/10.7202/1012481ar

Aller au sommaire du numéro

Éditeur(s)

Canadian Society for Eighteenth-Century Studies / Société canadienne d'étude du dix-huitième siècle

ISSN

1209-3696 (imprimé)

1927-8284 (numérique)

Découvrir la revue

Citer cet article

Senior, N. (1996). L'ange et le cadavre puant : Doctrine et culte des anges en France au XVIII ${ }^{\mathrm{e}}$ siècle. Lumen, 15, 185-194. https://doi.org/10.7202/1012481ar d'utilisation que vous pouvez consulter en ligne. 


\section{L'ange et le cadavre puant: Doctrine et culte des anges en France au XVIIIe siècle}

Les anges sont à la mode aujourd'hui, surtout en Amérique du nord. $A$ Book of Angels par Sophy Burnham (Ballantine, 1990) a été un best-seller, un autre de la même auteur a connu un succès comparable, et la même maison d'édition a toute une série sur les anges. On doit supposer que les éditeurs en sont aux anges. Le magazine Time a consacré un reportage au phénomène, avec portrait d'ange sur la couverture (27 décembre 1993), et Newsweek et d'autres magazines à grand tirage ont fait de même. Certaines librairies ont une section sur les anges, à côté de la santé et du jardinage.

Les anges étaient bien vivants au XVIIIe siècle aussi. Ils faisaient partie de l'univers de beaucoup de gens, probablement de la grande majorité de la population. Selon Robert Dawson, un grand succès de librairie en France, parmi les livres les plus imprimés tout au long du XVIIIe siècle, était L'Ange conducteur (l'ange gardien) par Jacques Coret. En comparaison avec cela, même des auteurs très connus comme Voltaire étaient lus par un petit cercle. Nous appelons le XVIIIe siècle le siècle des Lumières ; mais la lumière venait peut-être d'une source céleste. Je vais donc parler de certains livres destinés à un public dévot, c'est-à-dire à la plus grande partie du public lisant.

L'ange ou messager de Dieu appartient à une longue tradition. Le terme en hébreu est malak; en grec, aggelos. Ces termes s'emploient quelquefois pour un messager humain, quelquefois pour un être céleste. Je ne considère ici que les messagers de la divinité.

Au dix-huitième siècle ces porte-parole de la divinité paraissent dans les oeuvres de différents auteurs, notamment Voltaire. Ses anges les mieux connus sont ceux des contes philosophiques. Dans Zadig et dans Memnon, le personnage principal rencontre un ange (Zadig) ou génie (Memnon) qui lui explique le sens de ses aventures et de ses souffrances. L'explication n'a pas beaucoup de sens, car Voltaire ne prend pas ses anges au sérieux ; il joue avec les possibilités narratives et satiriques du personnage. ${ }^{1}$ 
Le Monde comme il va a une structure un peu différente. Babouc, bien que simple mortel, est envoyé par une divinité pour s'informer si la ville de Persépolis mérite la destruction - allusion évidente aux anges de la Genèse (Ch. 18 et 19) qui vont prendre ces mêmes renseignements sur Sodome et Gomorre. On voit donc l'action du point du vue de l'ange, bien que ce soit un ange souvent perplexe. Sa conclusion est différente de celle des anges de Sodome. Si tout n'est pas bien, tout est passable, et on laisse subsister Persépolis.

Mais au dix-huitième siècle, l'ange n'était pas simplement une figure de convention littéraire. C'était une réalité pour les théologiens, pour certains savants, et pour un public considérable qui pratiquait le culte des anges. Je vais donc parler brièvement de ces trois aspects : premièrement la tradition théologique et philosophique ; deuxièmement le culte des anges; et finalement l'érudition biblique.

L'Eglise a toujours enseigné l'existence des anges, mais le rôle qu'ils jouent a varié. Le quatrième concile de Latran a déclaré en 1215 que ce sont des êtres purement spirituels. C'est aussi une idée-clé du traité sur les anges dans la Summa de Saint Thomas d'Aquin (13e siècle), une oeuvre de base de l'angélologie. La dernière grande oeuvre de théologie scolastique sur les anges est le traité De angelis de Suarez, publié au milieu du XVIIe siècle, un livre de mille pages en latin, sans doute destiné à un public assez restreint.

Une question particulière est celle des rangs ou des ordres parmi les anges. La Hiérarchie céleste, qui semble venir du cinquième ou sixième siècle, était attribuée à Denys l'aréopagite, qui selon la légende avait été converti par Saint Paul. On croyait donc que c'était une oeuvre du premier siècle de notre ère. Ce traité fixe pour longtemps les idées sur les neuf ordres des anges, qui sont de bas en haut : anges, archanges, principautés; puissances, vertus, dominations ; trônes, chérubins, séraphins. Cette hiérarchie, qui consiste en trois fois trois, est une trinité de trinités, une sorte de trinité carrée. On peut être surpris de voir les archanges placés si bas; c'est que les anges des ordres plus hauts ne remplissent pas les fonctions de coursiers entre Dieu et les êtres humains.

L'auteur soutient ses théories par des raisons basées sur des passages bibliques et sur la logique, mais en même temps il reconnaît l'importance de l'imagination. Il explique que les descriptions bibliques d'anges, qui semblent leur donner des corps, sont métaphoriques. Dieu et les choses célestes sont au-dessus de notre compréhension, et 'il nous faut le secours d'un symbolisme proportionné à notre faiblesse' (La Hiérarchie céleste, trad. Darboy, 185). Ces symboles, malgré leurs défauts, nous sont utiles : 'Que ces défectueux rapprochements aident notre pensée à s'élever, c'est, je crois, ce qu'un homme réfléchi ne voudra pas nier' (187). La théologie chrétienne, selon Denys, enseigne les neuf appellations que 
nous venons de mentionner et qui sont le signe des propriétés qui caractérisent les ordres (203-204). C'est en contemplant ces natures toujours plus hautes, toujours plus parfaites, qu'on s'élève à la pensée de Dieu.

Au seizième siècle des érudits comme Laurent Valla et Erasme émettent des doutes sur l'authenticité de cette oeuvre, mais l'Eglise catholique la défend, et les neuf ordres sont encore importantes au dix-huitième siècle. $^{2}$

Les Protestants pour leur part reconnaissent l'existence des anges et les respectent, mais ils n'approuvent pas la prière aux anges. Au lieu de passer par une hiérarchie quelconque, pensent-ils, on doit s'adresser directement au créateur. On voit bien le parallèle entre cette idée et leur attitude envers la hiérarchie du clergé. Comme on le verra plus bas, les anges leur en veulent de ce manque d'égards.

Nous voyons donc deux courants dans la spéculation : d'un côté, ce qu'on peut déduire logiquement des premiers principes philosophiques ou théologiques, avec quelques passages bibliques bien choisis; de l'autre côté, ce qu'on peut trouver par l'imagination et la métaphore, toujours avec des passages bibliques choisis. Ces deux tendances ne sont pas tout à fait opposées; St. Thomas, qui veut tout prouver et tout intégrer dans un système, cite souvent le pseudo-Denys. Mais un auteur penche plutôt pour l'une ou pour l'autre tendance. C'est l'imagination et non la logique qui va jouer un rôle essentiel dans la dévotion.

La dévotion aux anges, comme la croyance à leur existence, est ancienne. L'auteur de l'épître aux Colossiens avertit ses lecteurs que tous les êtres célestes ont été créés par Dieu et lui sont donc subordonnés. Des déclarations semblables se trouvent dans l'épître aux Ephésiens (1.21) et dans le premier chapitre de l'épître aux Hébreux. La doctrine de la subordination des anges à Dieu et au Christ est souvent répétée au cours des siècles. Toutefois, à certaines époques la dévotion aux anges est encourageé par l'Eglise catholique, et elle a connu un grand essor au cours des XVIIe et XVIIIe siècles. C'était aussi le temps des confréries ou des associations en l'honneur des anges. ${ }^{3}$ Un des livres de dévotion les plus appréciés était la célèbre Introduction à la vie dévote par François de Sales (publié en 1608, avec de nombreuses éditions et réimpressions aux XVIIe et XVIIIe siècles). Sales mentionne souvent les anges, et ne semble pas faire de distinction bien nette entre la nature humaine et la nature angélique. Les êtres humains peuvent espérer ressembler aux anges ou même devenir anges eux-mêmes. L'échelle de Jacob offre l'image de la vie dévote: 'ce sont des hommes qui ont des coeurs angéliques, ou des anges qui ont des corps humains... Ils ont des ailes pour voler, et s'élancent en Dieu par la sainte oraison ; mais ils ont des pieds aussi pour 
cheminer avec les hommes par une sainte et aimable conversation... telles sont les personnes dévotes' (Première partie, 8-9).

L'emploi de l'imagination est la méthode principale de ce livre, et Sales donne des instructions spécifiques à sa lectrice Philothée: 'Imaginez-vous d'être en rase campagne toute seule avec votre bon ange, comme était le jeune Tobia allant en Ragès.' L'ange gardien montre, un jour, le paradis et ses délices ; un autre jour, l'enfer avec ses tourments. Le moyen de participer sans danger aux passe-temps obligatoires pour son état est de les voir du point de vue du ciel. 'Notre-Seigneur, NotreDame, les anges et les saints vous ont vue au bal. Ah! que vous leur avez fait grande pitié, voyant votre coeur amusé à une si grande niaiserie et attentif à cette fadaise!' (Troisième partie, Ch. XXXIII, 304)

Les anges de Sales sont hostiles aux protestants. Pierre Favre, en passant par des régions hérétiques de l'Allemagne, priait à l'ange responsable de chaque paroisse par laquelle il passait, et ces anges le garantissait des embûches des hérétiques (Deuxième partie, Ch. XVI, 118).

Si le livre de Sales a connu un grand succès, La Dévotion aux neuf choeurs des Saints Anges par Henry Marie Boudon (1668) ne semble pas avoir été si bien connue. Boudon a un goût décidé du paradoxe. Au lieu de montrer les anges comme nos compagnons, et l'état angélique comme une condition à laquelle nous pouvons aspirer, il insiste sur la distance entre les deux états. Une prière à l'ange gardien l'adresse comme 'Monseigneur, et fidèle guide de ma vie.' Cet être, 'pur esprit' et 'grand roi du paradis,' est infiniment supérieur à nous, car nous ne sommes 'que poussiere, \& que cendre, qu'un chetif morceau de bouë' (Dédicace 'A mon bon Ange Gardien').

Et pourtant, selon Boudon, 'les anges sont nos valets, \& nos esclaves, ô bonté de Dieu! des Princes du Paradis, des Rois de gloire \& nos valets, \& nos esclaves' (113). Ils s'abaissent jusqu'à nous servir de façon tout à fait pratique, paraît-il : 'ils vont quelquefois jusques à cét excez qu'ils gardent les bêtes pour l'amour des hommes... quelquefois déguisez en bergers, ils ont veillé sur des troupeaux de certaines âmes d'élite... (116). 'Ils font la fortune, \& enrichissent ceux qui les servent, quand cela n'est pas contraire à l'ordre de Dieu' (126).

Une remarque de Boudon explique peut-être l'hostilité des anges envers les Protestants. Des églises et des oratoires ont été consacrés à Dieu en l'honneur des anges, et les fidèles y font des pèlerinages. Or, 'Les Hérétiques à qui toute pitié est odieuse, blâment les Pelerinages que la sainte Eglise catholique approuve...' (402). Il paraît qu'un peu de ressentiment entre même dans de purs esprits célestes.

J'ai mentionné plus haut L'Ange conducteur de Jacques Coret (16311721). Coret, qui était jésuite, est l'auteur de plusieurs livres de dévotion, 
mais c'est $L^{\prime}$ Ange conducteur qui a connu le plus de succès. ${ }^{4}$ Le but de la dévotion à l'ange gardien était de se préparer pour une bonne mort ; à cette fin, on devenait membre d'une Association ou d'une Confrérie de l'Ange gardien, et on priait pour soi et pour les autres associés.

Comme Sales, Coret fait appel explicitement à l'imagination. 'Imaginez-vous que votre Ange vous dit : Mon cher Pupille, puisque Dieu vous donne encore cette journée, tâchez de la bien emploïer à l'aimer, le servir $\&$ le glorifier de votre mieux' (édition de 1746, 19). Comme on s'y attendrait, étant donné le but de l'Association, l'ange gardien est particulièrement consolant au moment de la mort. 'J'ai vu mourir beaucoup de personnes affectionnées à leur Ange, \& j'ai toûjours admiré l'extrême joye qu'elles goûtaient à la mort. Les uns me disoient : Dieu! que je suis heureux d'avoir aimé \& invoqué mon Ange. Dautres (sic) : je n'aurois jamais crû d'avoir le bien de mourir si content' (L'Association, Avis au lecteur).

Coret raconte des incidents où des anges gardiens sont intervenus dans la vie des particuliers pour les sauver de dangers tant spirituels que matériels. Par exemple, dans L'Association (1732) on apprend que L'Affard, gentilhomme allemand, négligeait depuis quelque temps de prier son ange. L'ange le prit par la main, le mena dans un cimetière, 'lui fit voir un cadavre puant \& hideux qu'il avoit d'un coup de pied fait sortir de terre,' et dit d'un air à le foudroyer, 'Tu sera (sic) tel.' Le pauvre homme se jeta aux pieds de l'ange, devint moine, et à sa mort était visiblement assisté par son ange et 'penetré de toutes les douceurs du Paradis.'

Les anges de Coret comme ceux de Sales et de Boudon sont anti-protestants. Un prêtre qui avait prié les anges pour la conversion d'un hérétique a été appelé au chevet du mourant. Un ange avait paru à l'homme et l'avait menacé de damnation éternel s'il mourait dans sa religion. L'homme terrifié a demandé au prêtre de brûler sa Bible et son exemplaire des hymnes de Marot.

A la différence de Boudon, qui se sert d'un langage respectueux dans les prières aux anges, Coret leur parle sur un ton d'intimité et d'affection, voire de passion. Les prières commencent souvent par des expressions comme : 'Mon ange gardien, le plus chéri de mon coeur...' 'O mon cher Ange! ô mon Prince! ô le cher conducteur de ma vie! je me jette entre vos bras, je me repose tranquillement en votre amour.' (édition de 1746, 299) 'O mon Ange, mon coeur ne suffit point pour vous aimer, il est trop petit, je voudrois en avoir des millions aussi grands que tout le monde pour vous aimer!' $(1746,177)$ L'ange semble jouer le rôle d'amant de coeur ; il offre une possibilité de tendresse sans danger et sans péché. L'auteur suggère de se procurer le portrait de l'ange : 'Pour marque que vous l'aimez, ayez en votre chambre une de ses Images, pour le saluer en entrant ou en sortant, le reconnoissant votre Maître, votre Pédagogue, 
votre Tuteur, votre Défenseur, votre Guide \& votre Garde très fidèle' $(1774,207)$.

Bien que ce livre soit dédié tout particulièrement à l'ange gardien, les neuf ordres d'anges sont présents aussi et on s'adresse à eux dans cette prière :

Séraphins ardens, embrasez-moi.

Chérubins très-sages, enseignez-moi.

Trônes suprêmes, purifiez-moi.

Dominations très-hautes, commandez-moi.

Vertus célestes, fortifiez-moi.

Puissances invincibles, défendez-moi.

Principautés souveraines, gouvernez-moi.

Archanges très-nobles, conduisez-moi.

Anges très-saints, gardez-moi, afin que je puisse servir, aimer, bénir \& glorifier avec vous la sainte Trinité, maintenant \& toûjours. Ainsi soit-il.

(édition de 1746, 304)

Jetons maintenant un coup d'oeil sur un exemple d'érudition biblique sur les anges. J'ai commencé par mentionner Voltaire; or, d'où viennent les connaissances de Voltaire sur la Bible? Elles viennent en grande partie de l'oeuvre d'Augustin Calmet, bénédictin très savant et auteur de nombreuses oeuvres sur la Bible. ${ }^{5}$ Même si Calmet n'a pas été si souvent réédité que Coret, parce que ses livres étaient très gros et très chers, il est bien connu et souvent cité tout au long du siècle.

Calmet discute les anges dans plusieurs livres : son Dictionnaire de la Bible, son Histoire universelle, et surtout son monumental Commentaire littéral sur la Bible. Ce commentaire contient une Dissertation sur les bons et les mauvais anges, ainsi que de nombreuses discussions éparses dans les notes sur différents livres de la Bible.

Calmet ne s'intéresse pas beaucoup aux possibilités de l'imagination au service de la religion. Il veut plutôt savoir d'où viennent les idées, et si elles sont vraies. C'est-à-dire qu'il fait deux sortes d'études : d'un côté l'histoire intellectuelle, où il trace les origines et l'évolution des idées ; de l'autre côté, l'exposition de la doctrine catholique. Quelquefois ces deux projets semblent impossibles à poursuivre en même temps.

Un exemple de Calmet comme détective biblique donnera un aperçu de sa méthode. Dans la Genèse, quand Dieu a chassé Adam et Eve du Paradis terrestre, il a placé un chérubin à l'entrée. Qu'est-ce que c'est qu'un chérubin? Selon Calmet, c'est un boeuf, ou plutôt c'est un animal composé où le boeuf a beaucoup de part. Ce n'était pas l'opinion acceptée à l'époque, mais Calmet l'explique par l'étymologie et par d'autres passages de la Bible (Commentaire littéral I, 35 et VI, 369). Or un 
siècle et demi plus tard, vers la fin du XIXe siècle, on a découvert, enterrées sous le sable, à l'entrée des palais assyriens, d'énormes sculptures de taureaux ailés au visage humain ... bref, des chérubins.

Calmet est aussi l'auteur d'un livre intitulé Dissertations sur les apparitions des anges, des démons et des esprits, qui a paru en 1746. On y trouve quelques-unes des mêmes préoccupations que dans ses grandes oeuvres. Partout il demande ce qui est vrai, ce qu'il faut croire littéralement. $\mathrm{Au}$ cours de son enquête, il raconte beaucoup d'anecdotes.

Quelquefois, paraît-il, les anges ou esprits sont doués pour les langues. Un tel esprit a apparu en rêve à un conseiller du Parlement et a prononcé quelques mots que l'homme n'a pas compris, mais qu'il a écrits en se réveillant. On lui a expliqué que c'était des mots syriaques écrits en français, et qu'ils voulaient dire 'sors de ta maison, car elle tombera en ruine aujourd'hui à neuf heures du soir.' Malgré la moquerie de quelques personnes, l'homme a déménagé, et 'la maison, depuis le comble jusqu'en bas, s'écroula à l'heure précise que ce charitable Génie lui avoit marquée.' Pourquoi donner l'avertissement dans une langue inconnue au destinataire? La raison, paraît-il, c'est que l'homme n'aurait pas pris au sérieux un message en français; il l'aurait attribué à l'imagination (60-61). Nous pouvons sans doute supposer que dans sa sagesse surnaturelle, l'ange savait que l'homme pouvait s'adresser à un érudit sachant le syriaque.

Encore faut-il s'assurer que les apparitions ont vraiment eu lieu. Calmet avait entendu dire qu'un certain M. Casmet à Toul avait un mouchoir sur lequel l'empreinte d'une main avait été faite par un esprit souffrant dans les flammes de l'enfer. Que fait un historien responsable? Il vérifie; et Calmet écrit à Toul pour s'informer sur cette histoire. Son correspondant répond que $\mathrm{M}$. Casmet affirme avoir le mouchoir quelque part, mais ne pas savoir où il est. Or, dit le correspondant, 'depuis j'ai appris que tout ceci est une fable, \& que l'empreinte de la main sur le mouchoir avoit été faite par un Garçon Maréchal, avec un fer chaud fait en forme de main, pour mieux jouer son jeu \& entretenir son commerce de galanterie avec la Demoiselle'(122-125).

Il y a donc, selon Calmet, beaucoup de fraudes dans le domaine des apparitions, et il y a un autre danger aussi. Souvent les prétendues apparitions d'anges ou d'esprits sont des pièges du démon, qui veut que les pécheurs continuent à vivre dans l'impénitence, comptant sur la possibilité de se repentir avant la mort (131-132).

On voit donc que Calmet est en même temps croyant et sceptique, esprit crédule et historien désabusé.

L'image de l'ange au XVIIIe siècle est très riche ; les écrits vont de l'analyse érudite à la dévotion sentimentale. Manifestation d'un Dieu trop éloigné de la finitude humaine, l'ange est plus facile à concevoir et 
donc plus rassurant que son Créateur. L'ange gardien, qui est en même temps grand seigneur céleste et notre ami ou même notre valet, plaît particulièrement à l'amour-propre humain. L'ange comme il paraît dans les livres de dévotion est un élément important dans l'imaginaire d'un siècle souvent considéré comme sceptique.

En même temps Calmet, également dévot mais aussi homme des Lumières à bien des égards, pose la question de l'origine de ces idées, et surtout la question : les apparitions des anges sont-elles authentiques ou non? Les auteurs de livres de dévotion aux anges racontent des anecdotes édifiantes ; cela ne suffit pas pour Calmet. Les anges, et les démons, sont importants, justement ; ils sont trop importants pour qu' on les laisse à l'imagination. Il faut savoir la vérité.

\section{NANCY SENIOR \\ University of Saskatchewan}

\section{Notes}

1 Dans Candide, Voltaire se passe de ce symbole de la transcendence. La fonction de l'ange est partagée par deux personnages humains : le derviche qui explique qu'on ne peut rien comprendre, et le vieux Turc qui donne des conseils sur la bonne manière de vivre. Dans Micromégas, on pourrait dire que les deux voyageurs de l'espace ont quelques caractéristiques angéliques, malgré leur présence physique indéniable. Ils viennent du ciel, ils savent plus que les êtres humains (mais pas tout), et leur message final, comme celui des anges de Zadig et de Memnon, est incompréhensible. Pour les liens entre anges et extraterrestres, voyez le livre de Jung.

2 Voyez l'introduction de René Roques à La Hiérarchie céleste, 1970.

3 L'Association ou la bonne mort de Coret (1732) tire son titre de l'Association, qui est 'une union de prières adressées aux Anges par plusieurs personnes qui veulent s'obtenir les uns aux autres une sainte mort.' L'Association est mentionnée aussi dans l'édition de 1750 de L'Ange conducteur. Il n'est pas clair quel est le rapport entre cette Association et la Confrérie des saints Anges Gardiens (éd. de 1746, 304), ou la Confrairie de l'Ange-Gardien (éds. de 1750 et de 1774, mentionnée dans le titre complet). Voyez les articles sur les anges par Joseph Duhr dans le Dictionnaire de spiritualité, ascétique et mystique, doctrine et histoire, 1937.

4 Au cours du dix-huitième siècle L'ange conducteur a eu beaucoup d'éditions assez différentes les unes des autres, de sorte qu'il n'est pas facile de savoir quelles parties sont de Coret et lesquelles ont été ajoutées par d'autres auteurs ou éditeurs. Je vais parler comme si Coret était l'auteur de tout, et mes exemples sont 
tirés de différentes éditions. L'Association ou la bonne mort contient à peu près les mêmes idées que L'Ange conducteur.

5 Voyez N. Senior, 'Angels, demons and dom Calmet,' Studies on Voltaire and the Eighteenth Century, 332: 155-188.

\section{Ouvrages cités}

\section{Sources primaires:}

Boudon, Henry Marie. La Dévotion aux neuf choeurs des saints anges. Paris, 1668.

Calmet, Augustin. Commentaire littéral sur tous les livres de l'Ancien et du Nouveau Testament. 8 t., Paris, $1724-1726$.

Calmet, Augustin. Dictionnaire historique, écritique, chronologique, ge-graphique et littral de la Bible. 4 t., 1722.

Calmet, Augustin. Dissertations sur les Apparitions des Anges, des Démons et des Esprits, et sur les Revenans et Vampires De Hongrie, de Bohème, de Moravie E de Silésie. Paris, 1746.

Calmet, Augustin. Histoire universelle, sacrée et profane, depuis le commencement du monde jusqu'à nos jours. 6 t. Strasbourg, 1735.

Calmet, Augustin. Histoire de la vie et des miracles de Jésus-Christ; tirée de l'histoire de l'Ancien $\mathcal{E} d u$ Nouveau Testament... Paris, 1720.

Coret, Jacques. L'ange conducteur dans la dévotion chrétienne, réduite en pratique en faveur des ames dévotes... Liège, 1746.

Coret, Jacques. L'Ange conducteur dans la dévotion chrétienne réduite en pratique en faveur des ames dévotes.... Nancy, 1750.

[Coret, Jacques] L'Ange conducteur dans la dévotion chrétienne, réduite en pratique en faveur des ames dévotes... Lille, 1774.

Coret. L'Association ou la bonne mort sous la protection des Saints Anges gardiens. Saint Omer, 1732.

Denys l'Aréopagite (le pseudo-Denys, Dionysius the Areopagite, the pseudoDionysius). La Hiérarchie céleste. Introduction par René Roques, étude et texte critique par Gunther Heil, traduction et notes par Maurice de Gandillac. Paris: Les Editions du Cerf, 1970.

Denys l'Aréopagite. Oeuvres de Saint Denys l'Aréopagite, traduites du grec par l'abbé Darboy. Paris: Sagnier et Bray, 1845.

Sales, François de. Introduction à la vie dévote. Paris: Société typographique, 1804 (1st ed. 1608).

\section{Sources secondaires:}

Adler, Mortimer. The Angels and Us. New York: Collier, 1982. 
Ages, Arnold. 'Voltaire, Calmet and the Old Testament.' Studies on Voltaire and the eighteenth century, 41 (1966), 87 - 187.

Anchor Bible Dictionary, The. David N. Freedman, ed. New York: Doubleday, 1992. Articles Angels, Cherubim.

Belaval, Yvon et Dominique Bourel, eds. Le Siècle de Lumières et la Bible. Paris: Beauchesne, 1986.

Bessire, François. 'Voltaire lecteur de dom Calmet,' Studies on Voltaire and the eighteenth century, 284 (1991), 139-177.

Digot, Auguste. Notice biographique et littéraire sur Dom Augustin Calmet. Nancy, 1860.

Duhr, Joseph. Articles Ange, Ange gardien, La dévotion aux anges, etc. dans Viller, Marcel, S.J., ed., Dictionnaire de spiritualité, ascétique et mystique, doctrine et histoire, t. 1, 1937.

Jung, C. G. Flying Saucers: A Modern Myth of Things Seen in the Skies. Translated by R. F. Hull. Princeton, N.J.: Princeton University Press, 1991.

Ries, Julien et Henri Limet, eds. Anges et démons.. Actes du colloque de Liège et de Louvainla-Neuve, 25-26 novembre 1987, Homo religiosus 14. Louvain-la-Neuve: Centre d'Histoire des religions, 1989.

Tavard, Georges. Les Anges. Traduit de l'allemand par Maurice Levèvre. Histoire des dogmes t. II, fascicule 2b. Paris: Les Editions du cerf, 1971.

Thompson, Keith. Angels and Aliens. UFOs and the Mythic Imagination. Reading, Mass: Addison-Wesley, 1991. 